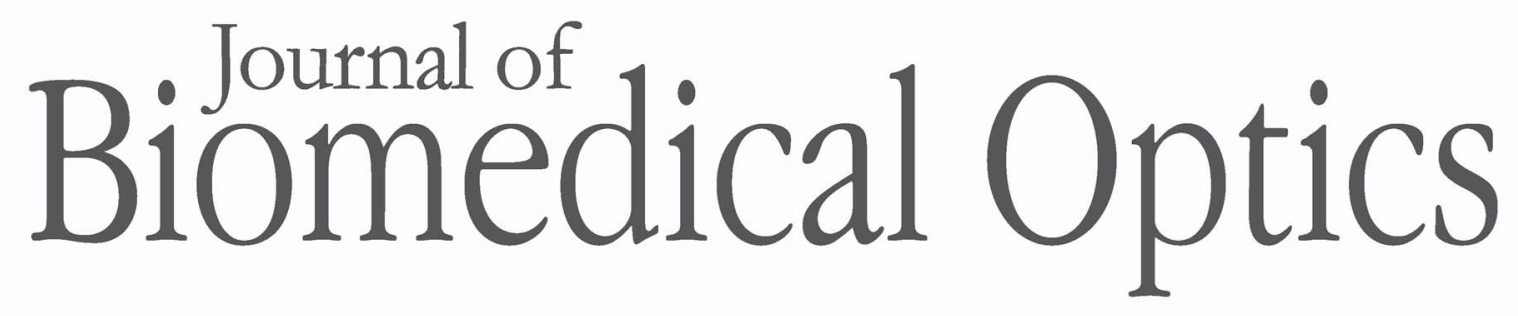

\title{
Estimating the density of fluorescent nanoparticles in the primo vessels in the fourth ventricle and the spinal cord of a rat
}

Jaekwan Lim

Jong Hyun Jung

Sungwoo Lee

Zhendong Su

Zhou Qiang

Jin-Myung Cha

Jin-Kyu Lee

Kwang-Sup Soh 


\title{
Estimating the density of fluorescent nanoparticles in the primo vessels in the fourth ventricle and the spinal cord of a rat
}

\author{
Jaekwan Lim, ${ }^{a}$ Jong Hyun Jung, ${ }^{a}$ Sungwoo Lee, ${ }^{a}$ Zhendong Su, ${ }^{a}$ Zhou Qiang, ${ }^{a}$ Jin-Myung Cha, \\ Jin-Kyu Lee, ${ }^{b}$ and Kwang-Sup Soh ${ }^{a}$ \\ ${ }^{a}$ Seoul National University, Nano Primo Research Center, Advanced Institutes of Convergence Technology, \\ 864-1 lui-dong, Yeongtong-gu, Suwon-si, Gyeonggi-do, Republic of Korea 443-270 \\ bSeoul National University, Materials Chemistry Laboratory, Room 506, Bldg. 503, Kwanak-gu, Seoul, \\ Republic of Korea 151-747
}

\begin{abstract}
The primo vascular system is a novel circulatory system forming a network throughout an animal's body. Primo vessels were recently observed in the fourth ventricle of the brain and in the spinal cord of a rat by using fluorescent nanoparticles. In order to quantify the nanoparticles in the primo vessels, we measured the florescence of the nanoparticles and calibrated the measurements by using a reference suspension. We removed the noise due to autofluorescence with the technique of multispectral imaging. The line densities of nanoparticles and the contrast values of their images were, respectively, $0.5 \pm 0.5 \mathrm{ng} / \mathrm{mm}$ and $0.7 \pm 0.5$ for primo vessels in the fourth ventricle, and $1.3 \pm 0.6 \mathrm{ng} / \mathrm{mm}$ and $1.4 \pm 0.2$ for primo vessels in the spinal cord. The data obtained from and the procedures used in this work could be useful in evaluating the feasibility of using nanoparticles as a contrast agent during MRI or CT imaging of primo vessels in the brain or the spinal cord. $\odot 2011$ Society of Photo-Optical Instrumentation Engineers (SPIE). [DOI: 10.1117/1.3647595]
\end{abstract}

Keywords: brain; spinal cord; primo vessel; primo vascular system; cerebrospinal fluid; nanoparticle; fluorescence.

Paper 11235RR received May 19, 2011; revised manuscript received Sep. 15, 2011; accepted for publication Sep. 19, 2011 ; published online Oct. 27, 2011.

\section{Introduction}

The primo vascular system (PVS) is a novel circulatory system distributed throughout an animal's body. It was first proposed by $\mathrm{Kim}^{1,2}$ in the early 1960 s as the anatomical substance of acupuncture meridians, but until recently ${ }^{3}$ his claim was not confirmed except for a partial reproduction by the Japanese anatomist Fujiwara. ${ }^{4}$ The recent confirmation was possible because the methods necessary to detect and identify the PVS have been developed and allowed the PVS to be observed in various organs of an animal's body.

The PVS was observed in circulatory systems, that is, inside blood vessels ${ }^{5}$ and lymph vessels ${ }^{6,7}$ of rabbits, rats, and mice. Reassuringly, its presence in a large animal was also verified when the PVS was observed in the bovine heart. ${ }^{8}$ The PVS lying on the surfaces of internal organs, ${ }^{9}$ like the liver, stomach, intestines, bladder, and others was intensively studied because it was rather easily detected in the abdominal cavity. More recently, the PVS was observed floating in the cerebrospinal fluid (CSF) of the fourth ventricle and in the central canal of the spinal cord of a rabbit by using a hematoxylin staining technique after brain surgery. ${ }^{10}$ Subsequently, the detection method was improved to inject nanoparticles into the lateral ventricle of a rat before surgery. ${ }^{11}$

Some of the functional aspects of the PVS were investigated. First of all, the flow speed of fluid in the primo vessels on the

Address all correspondence to: Seoul National University, School of Physics, Biomedical Physics Lab., San 56-1, Sillim-Dong Kwanak-Gu, Seoul, Seoul, 151747 Republic of Korea; Tel: 82-2-8807750; Fax: 82-2-7697; E-mail: kssoh@snu.ac.kr. surfaces of the internal organs of rabbits was measured to be $0.3 \mathrm{~mm} / \mathrm{s},{ }^{12}$ which is much slower than the flow speed of blood or lymph. One important component in the PVS fluid claimed by Kim was the neurotransmitter hormone catecholamine. ${ }^{13}$ Its presence was indeed verified by measurements using the ELISA technique. Proteomic analysis ${ }^{14}$ of the primo fluid in the PVS of rabbits identified proteins related to carbohydrate-based processes. These proteins are known to be associated with mesenchymal stem cells or cancer cells.

About the medical relevance of the PVS, its presence around xenografted tumor tissues has raised a host of topics on the role of the PVS with respect to cancer biology and medicine. In particular, it was shown that the PVS might be an additional metastasis path for cancer cells. ${ }^{15,16}$ As another aspect, the primo fluid carries DNA-containing granules called primo microcells, ${ }^{17}$ or sanals as Kim named them. The size and the shape of sanals were similar to those of the very small embryonic-like stem cells ${ }^{18}$ which suggests the medical significance of the PVS in connection with regeneration. Kim claimed that the main function of sanals was regeneration of damaged organs, which awaits thorough investigation.

In order to elucidate the functional and the medical aspects of the PVS, an imaging system for observing the PVS in vivo in situ without surgery needs to be developed. The current work is the first step toward this eventual goal of imaging the PVS with MRI or other imaging modalities. For this purpose, contrast agents must be found and methods to inject them into the PVS must be developed. In this study, we used fluorescent nanoparticles,

1083-3668/2011/16(11)/116010/7/\$25.00 @ 2011 SPIE 
which were used in previous PVS research and were preferentially absorbed by the primo vessels compared with surrounding tissues. These nanoparticles were detectable with MRI. ${ }^{19}$ At the present time, there is no known method to inject contrast agents exclusively into the PVS. Thus, we injected the nanoparticles into the lateral ventricles of rats where the CSF flowed. The nanoparticles in the CSF flowed to the fourth ventricle and the central canal of the spinal cord and were absorbed by the primo vessel floating in the CSF. Current experiments were only primitive trials, but demonstrated that nanoparticles could be used as contrast agents. Estimating the density of the nanoparticles absorbed by the PVS is desirable in order to plan and evaluate the feasibility of future experiments on using MRI to image the PVS. ${ }^{20}$ In the present work, we attempted to estimate the absorbed nanoparticle density by using the fluorescence intensity and established quantification techniques.

\section{Materials and Methods}

\subsection{Animals}

Twenty male and female Sprague-Dawley rats (270 to $290 \mathrm{~g}$ ) were obtained from the Jung Ang Laboratory Animal Company (Seoul, Korea). The animals were housed in a temperaturecontrolled environment at $23{ }^{\circ} \mathrm{C}$ with $60 \%$ relative humidity. All animals were exposed to a 12-h light-dark cycle and had ad-libitum access to food and water. Procedures involving the animals and their care conformed to the institutional guidelines of Seoul National University, which were in full compliance with current laws and policies. ${ }^{21}$

\subsection{Fluorescent Tracer Suspension}

Cobalt-ferrite magnetic nanoparticles embedded in amorphous silica shells were synthesized. The shells contained a fluorescent dye, rhodamine B isothiocyanate (orange, $\gamma_{\max }=555 \mathrm{~nm}$ ), in the middle and a biocompatible polyethylene glycol on the outside. The average core size of the fluorescent magnetic nanoparticles was $9 \mathrm{~nm}$, while the average size of the enclosing shell was $50 \mathrm{~nm}$. The concentration of particles, which were suspended in a sterile saline solution at $p \mathrm{H} 7.4$, was $10 \mathrm{mg} / \mathrm{ml}^{22}$ The fluorescent tracer suspension was composed of fluorescent magnetic nanoparticles and $\geq 99.9 \%$ ethyl alcohol (Sigma, St. Louis, Missouri) in a ratio of 1:1.

\subsection{Stereotaxic Injection and Sample Preparation}

A stereotaxic system was used to inject the fluorescent nanoparticles (FNPs) precisely into the lateral ventricle. The rats were anesthetized with an intramuscular injection of urethane $(1.5 \mathrm{~g} / \mathrm{kg})$, and all surgical procedures were performed under general anesthesia. The rat's scalp was removed and a hole (diameter $=1 \mathrm{~mm}$ ) was drilled in the skull to access the brain. The hole had the stereotaxic coordinates of $2.0 \mathrm{~mm}$ after the bregma, $2.0 \mathrm{~mm}$ to the right of the middle line, and $4 \mathrm{~mm}$ in depth.

FNPs $(0.3 \mathrm{ml})$ were injected into the lateral ventricle by using a stereotaxic system with an injection needle (17-gauge syringe, $1.47 \mathrm{~mm}$ in diameter). Two hours later, the rat was decapitated, and the whole spine was isolated. The whole spine was immediately put into $0.4 \%$ neutral buffered formalin and kept in it for $24 \mathrm{~h}$. After $24 \mathrm{~h}$, the bone was removed from the spine, and the spinal cord was carefully exposed. After the entire spinal cord had been exposed, we measured the signal from the FNPs.

\subsection{Imaging Device and Image Processing}

A fluorescent stereomicroscope (MVX 10; Olympus Co., Tokyo, Japan) equipped with a sensitive black/white charged-coupled device camera and a long-pass filter $(600 \mathrm{~nm}$; CVI, Seoul, Korea) was used to detect the fluorescence from the nanoparticles. ${ }^{23}$ A sonicator (Digital Sonifier 450, Branson Co., Danbury, Connecticut) was used for breaking aggregates of nanoparticles in the suspension. Gray-scale images were analyzed using IMAGEJ software (Wayne Rasband, National Institute of Health, $\mathrm{v} 1.43 \mathrm{u}$ ). Gnuplot 4.4 was used to fit the functions.

\subsection{Calibration Material}

Reference suspensions for calibration were made to quantify the line densities of the nanoparticles in primo vessels. In order to avoid the formation of aggregates of nanoparticles, we applied sonication for about a minute. The densities of the reference suspensions were 50, 20,10,1,0.1, and $0 \mu \mathrm{g} / \mathrm{ml}$. Three (3.0) or $2.7 \mathrm{ml}$ of each reference suspension were poured into a small Petri dish whose diameter was $35 \mathrm{~mm}$ [Fig. 1(a)]. In order to reduce the background fluorescence, we placed the Petri dish on a piece of black leather. The same fluorescence filter was used to view the nanoparticles. Two fluorescent images of the reference suspension were taken for each density. The exposure times were from $250 \mathrm{~ms}$ to $12 \mathrm{~s}$ and were adjusted to obtain appropriate fluorescence intensities.

\subsection{Optical Contrast Measurement}

The Weber contrast ${ }^{24}$ is defined as $\left(I_{m}-I_{b}\right) / I_{b}$. Here, $I_{m}$ means the intensity of a measured point, and $I_{b}$ is the background intensity around the measured point. The larger the contrast, the more clearly we can see the image.

To measure the optical contrast of a fluorescent line, we measured the highest intensity over a specific part of the line and compared that value to the background. We analyzed the stereoscopic fluorescence images with bright fluorescent lines. We used IMAGEJ $1.44 \mathrm{c}$ software with the region of interest (ROI) manager function to analyze the images. First, we chose places to be analyzed. The brightness of a line varied over its length. Since several aggregates of FNPs made conspicuously bright spots, we avoided such spots. Second, we measured the background intensity. We chose five imaginary ovals near the fluorescent line as ROIs, and their mean intensities, $I_{b}$, were measured. Third, we measured the line intensity. Five imaginary rectangles covering the fluorescent line in the chosen place were drawn as ROIs. We chose the maximum value of the intensities in the ROIs as $I_{m}$. Fourth, we calculated the contrast for each ROI, the means, and the standard errors of the means. 
(a)
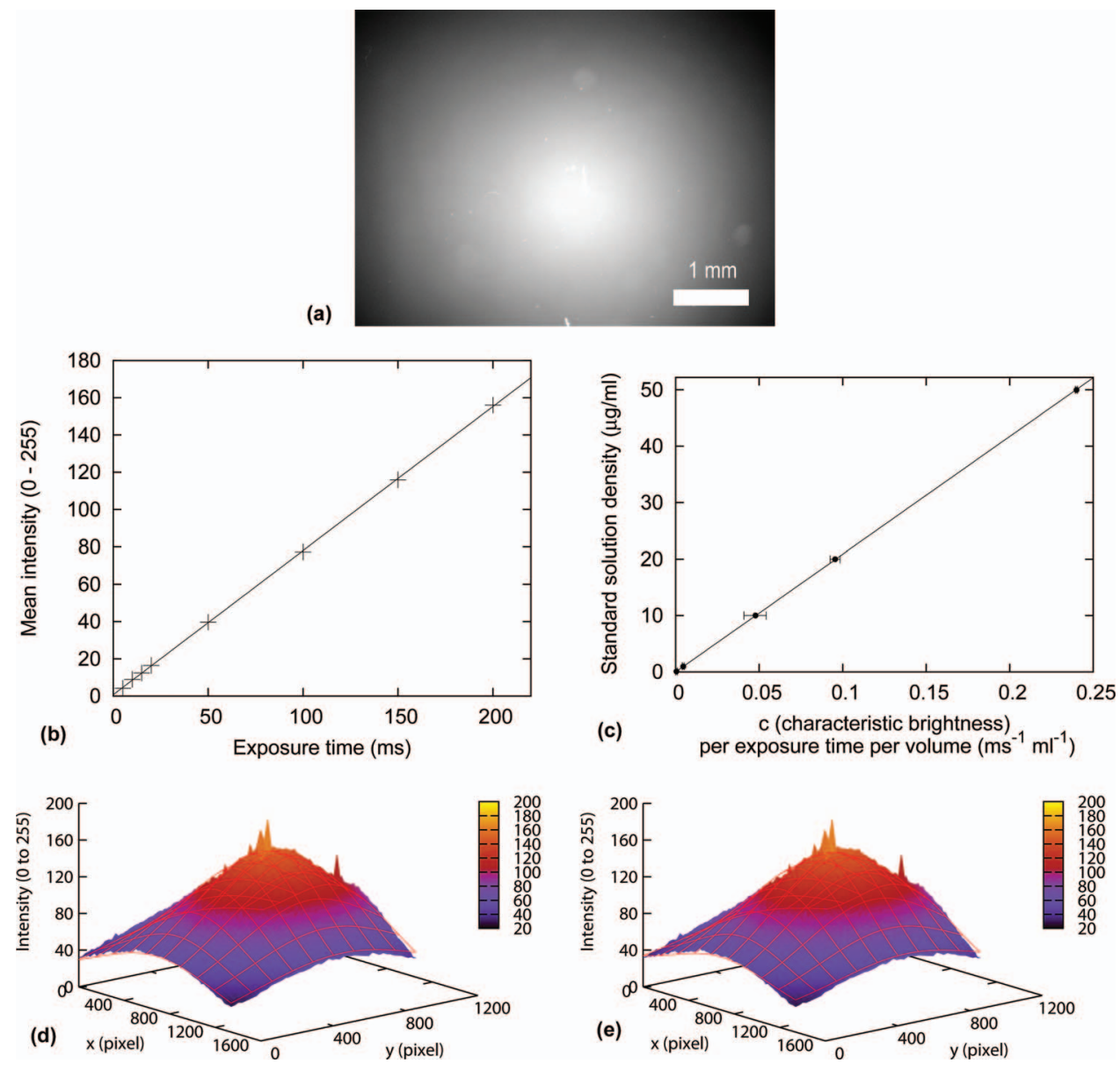

Fig. 1 (a) Inhomogeneous illumination of the nanoparticle suspension under a microscope. (b) Image intensity or brightness versus exposure time for the reference solution. The intensity of a sample is proportional to the exposure time. (c) Reference solution density versus brightness (intensity). The reference solution density is proportional to the brightness of an image. The $c$ value was calculated by using a Lorentzian model fitting. (d) The Lorentzian model fitting. (e) The Gaussian model fitting. Both models fit the data within $2 \%$ accuracy with respect to the root-mean-square residual.

\subsection{Multispectral Imaging for Removal of Autofluorescence}

To ensure that the sample signal was from the nanoparticles and not from autofluorescence, we used multispectral imaging techniques to remove the autofluorescence. Usually, the frequency range of autofluorescence is broad enough to encompass the whole visible light range. In contrast, a nanoparticle emits more red fluorescence than green and blue fluorescence. Thus, if red, green, and blue filters are used to take images, the light signals from autofluorescence and from the nanoparticles can be differentiated. Filters were used with a black-and-white CCD and all images were in gray scale (black and white correspond to 0 and 255 , respectively.). We followed a multistep process to remove the background noise due to autofluorescence. First, we evaluated the mean intensity of the background of an image for red, green, and blue filters. The intensity under the blue filter was negligible. Second, we calculated the ratio of the mean intensity of an image taken under the red filter to that taken under a green filter by using the ratio $b=$ (mean intensity of background under the red filter)/(mean intensity of background under the green filter). Third, we multiplied the intensity value at each pixel under a green filter by $b$ which was the effective red intensity due to autofluorescence. Fourth, we subtracted this effective red intensity of the background from the intensity of the image under a red filter at each pixel because the latter had contributions from both the nanoparticles and autofluorescence. In the resulting image [Fig. 3(c)], most of the background signal was removed, and we obtained a high contrast image of a primo vessel.

\section{Results}

The primo vessel in the fourth ventricle was transparent and not detectable with a stereomicroscope, but was detected with the fluorescence microscope due to the FNPs. It was not attached to the wall of the ventricle, but was a freely-floating structure in the CSF. As illustrated in Fig. 2(a), the primo vessel located in the fourth ventricle of the rat brain is a thin threadlike structure [arrow in Fig. 2(b)] that shows a strong fluorescence signal due to the nanoparticles that were absorbed by the primo vessel floating in the CSF. The average thickness of the primo vessel was $23 \pm 4 \mu \mathrm{m}$, and the average line density of the absorbed nanoparticles was $0.5 \pm 0.5 \mathrm{ng} / \mathrm{mm}$ (standard error) for the five rats. There were large variations in the absorbed nanoparticle densities for the subject rats. The average contrast was $0.7 \pm 0.5$, and in favorable cases, the contrast reached values 


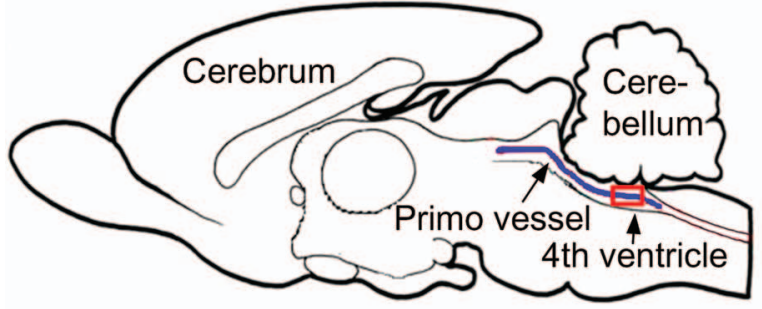

(a) (b)

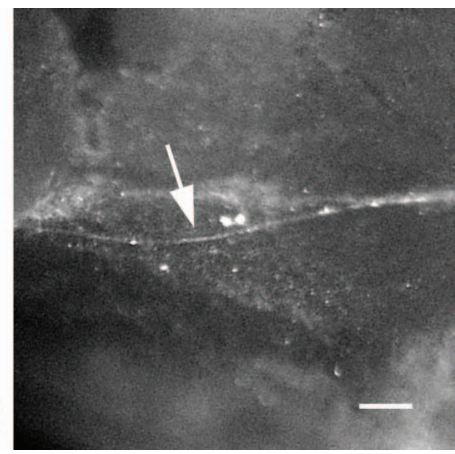

Fig. 2 Primo vessel in the fourth ventricle. (a) Illustration of the locations of the primo vessel samples in the fourth ventricle. (b) Picture of a thin primo vessel (arrow) in the fourth ventricle, as visualized using nanoparticles. The line density of nanoparticles in the primo vessels was quantified, and the contrast was calculated. The scale bar is $200 \mathrm{~nm}$.

higher than 1 (Table 1). When a sample's nanoparticle density was high, its image contrast was also high.

The illustration of Fig. 3(a) indicates the location of a primo vessel accompanying a blood vessel in the subarachnoid space of the spinal cord, and Fig. 3(b) shows the unprocessed image of the primo vessel (arrow) accompanying the blood vessel (broken arrow). Using image processing, we could remove the noise signals of the autofluorescence and obtain a clean signal from the primo vessel (arrow) beside the blood vessel [Fig. 3(c)].

Unlike the primo vessel floating in the ventricle, this one was fixed close to and along the blood vessel. The existence of such a primo vessel was not noticeable with an optical microscope without fluorescence. The thickness of the primo vessel was 19 $\pm 4 \mu \mathrm{m}$ (standard error), and that of the blood vessel was 68 $\pm 14 \mu \mathrm{m}$ (standard error). The average line density for the absorbed nanoparticles of four different rats was $1.3 \pm 0.6$ $\mathrm{ng} / \mathrm{mm}$, and the average contrast was $1.4 \pm 0.2$ (Table 2). The densities in the subarachnoid space were about 3 times higher than those in the fourth ventricle. The reason for this large difference in nanoparticle densities is not known.

\section{Analysis}

Analyzing the data from fluorescence images, we calculated the line densities of absorbed nanoparticles. For this purpose, we prepared reference suspensions and used Gaussian and
Lorentzian models to fit the intensity data to the given nanoparticle densities of the suspensions. With this calibration, we were able to obtain the line densities of the absorbed FNPs.

\subsection{Calibration with Reference Suspensions of FNPs}

Nanoparticles can be quantified by labeling with fluorescent, magnetic, or radioactive materials, ${ }^{26}$ and multispectral imaging can be used to differentiate a target from the background. ${ }^{27}$ Quantification of a photosensitizer, such as mesotetrahydroxyphenylchlorin, can be improved by considering the fluorescence of the background. ${ }^{28}$ The concentration of fluorophores in vivo at the site of photodynamic therapy has been estimated through a comparison with a calibrated measurement or a standard solution. ${ }^{29,30}$

In order to compare the density of FNPs in the primo vessel with that of the reference suspension, we took the effects of background, exposure time, and light sources into account. First, the background effect was considered by subtracting the intensity of a control suspension with $0 \mu \mathrm{g} / \mathrm{ml}$ of nanoparticles. The source of the background was mainly the fluorescence of the leather. Second, the effect of exposure time was considered. The exposure time was changed from 5 to $200 \mathrm{~ms}$, and the mean intensity was measured. The intensity was shown to be proportional to the exposure time $(t)$ [Fig. 1(b)]. If the reference suspension's exposure time was $t_{1}$ and if the control's exposure time was

Table 1 Line densities of the nanoparticles and contrasts of the images of primo vessels in the fourth ventricles of rats. Sampling areas were five adjoining regions in the sample images. All line densities and contrasts are averages \pm standard deviations.

\begin{tabular}{lccc}
\hline Primo-vessel sample & Thickness of primo Vessel $(\mu \mathrm{m})$ & Nanoparticle line density $(\mathrm{ng} / \mathrm{mm})$ & Contrast \\
\hline 1 & 24 & $0.13 \pm 0.03$ & $0.29 \pm 0.05$ \\
2 & 15 & $0.19 \pm 0.05$ & $0.50 \pm 0.09$ \\
3 & 15 & $0.04 \pm 0.01$ & $0.18 \pm 0.04$ \\
4 & 34 & $1.00 \pm 0.12$ & $1.39 \pm 0.17$ \\
5 & 24 & $1.04 \pm 0.08$ & $1.14 \pm 0.03$ \\
Average & $23 \pm 4$ & $0.5 \pm 0.5$ & $0.7 \pm 0.5$ \\
\hline
\end{tabular}




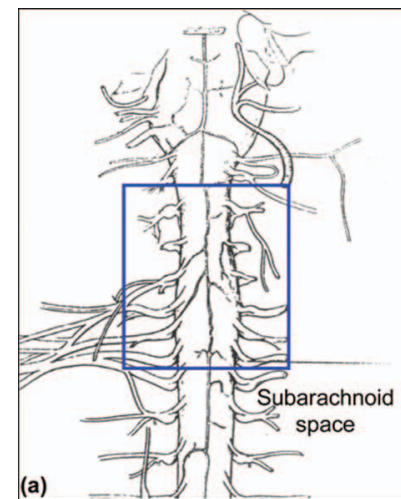

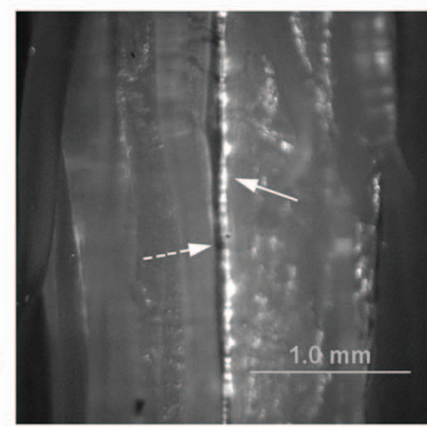

(b)

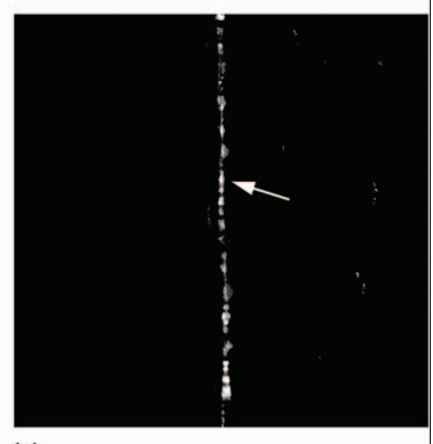

(c)

Fig. 3 Primo vessel in the spinal cord. (a) Illustration of the location of a vein just beside a primo vessel (the primo vessel is not shown in the illustration) in the subarachnoid space of the spinal cord. The illustration was modified from a reference book (Ref. 25). (b) Unprocessed picture of a primo vessel (arrow) accompanying a blood vessel (broken arrow) in the subarachnoid space of the dorsal surface of the spinal cord. The line density of nanoparticles in this sample was quantified. (c) The nanoparticle image of (b) after image processing to show only the nanoparticle signal by subtracting the background noise. The average contrast was 1.4 .

$t_{0}$, then the intensity of the control image was multiplied by $t_{1} / t_{0}$, and the adjusted control image was subtracted from the reference suspension's image. Third, the inhomogeneity of the illumination was taken into account because the center area of the reference suspension was more brightly illuminated than the peripheral area [Fig. 1(a)]. For this purpose, we used a shading correction without model functions $s^{31,32}$ or a second order polynomial function ${ }^{33}$ modeling the background image. Considering the intensity of the peak at the center which decreased toward the boundary, we tried Lorentzian and Gaussian fittings, instead of polynomial modeling, of the illumination intensity:

$$
I_{L}(r)=c\left[c_{0}+1 /\left(1+r^{2}\right)\right]
$$

and

$$
I_{G}(r)=c\left[c_{0}+\exp \left(-r^{2}\right)\right],
$$

where $I_{L}(r)$ and $I_{G}(r)$ are Lorentzin and Gaussian fitting intensities, respectively. Here, the dimensionless relative distance from the center is defined as:

$$
r(x, y)=\sqrt{\left(\frac{x-x_{m}}{\Gamma_{x} / 2}\right)^{2}+\left(\frac{y-y_{m}}{\Gamma_{y} / 2}\right)^{2}}
$$

where $x_{m}$ is the $x$ (pixel) value of the brightest point, and $y_{m}$ is the $y$ (pixel) value of the brightest point, and $\Gamma_{x}$ (pixel) and $\Gamma_{y}$ (pixel) are the full widths at half maximum intensity. The Lorentzian intensity $I_{L}$ and the Gaussian intensity $I_{G}$ were adapted from those used by Lettington et al. ${ }^{34}$ and Condon. ${ }^{35}$ The parameter $c_{o}$ was introduced to account for the deviation from a pure Lorentzian or Gaussian distribution. Thus, the parameters $c_{o}$, $\left(x_{m}, y_{m}\right)$, and $\left(\Gamma_{x}, \Gamma_{y}\right)$ describe the shape of illumination, and the overall factor $c$ represents the peak intensity at $\left(x_{m}, y_{m}\right)$. Both Lorentzian and Gaussian fittings turned out to be accurate enough. The root-mean-square residuals (RMSRs) were 3.33 [Fig. 1(d)] and 3.48 [Fig. 1(e)] for the Lorentzian and Gaussian, respectively. The degree of freedom was 3530 , and the theoretical maximum RMSR value was 255 . Therefore, our fittings were good to within a $2 \%$ accuracy. Fourth, a calibration of the nanoparticle density with fluorescence intensity was performed. The parameters $c_{o},\left(x_{m}, y_{m}\right)$, and $\left(\Gamma_{x}, \Gamma_{y}\right)$ were determined by fitting the intensity curve $I_{G}(r)$ or $I_{L}(r)$ to the reference suspension of highest density. The overall factors, $c$, representing the brightnesses of reference suspensions of various FNP densities were determined by using the intensity at $r=0$.

As demonstrated in Fig. 1(c), the density of FNPs in the reference suspensions and their brightnesses $c$ had a linear relation: $\rho V=a(c / t)$, where $V$ is the volume of the reference suspension,

Table 2 Line densities of the nanoparticles and the contrasts of the images of primo vessels on the spinal cords of rats. Sampling areas were five adjoining regions in the sample pictures. All line densities and contrasts are averages \pm standard deviations.

\begin{tabular}{lccc}
\hline Primo-vessel sample & Thickness of primo vessel $(\mu \mathrm{m})$ & Nanoparticle line density $(\mathrm{ng} / \mathrm{mm})$ & Contrast \\
\hline 1 & 20 & $1.8 \pm 0.2$ & $1.63 \pm 0.04$ \\
2 & 30 & $1.04 \pm 0.08$ & $1.20 \pm 0.04$ \\
3 & 10 & $0.53 \pm 0.13$ & $1.19 \pm 0.05$ \\
4 & 15 & $1.74 \pm 0.18$ & $1.68 \pm 0.02$ \\
Average & $19 \pm 4$ & $1.3 \pm 0.6$ & $1.4 \pm 0.2$ \\
\hline
\end{tabular}


$t$ is the exposure time, and $a$ is the slope of the linear relation. The coefficient $a$ was determined by using a linear fitting of the line in Fig. 1(c).

As an illustrative example, for a reference suspension, the parameters fitting the brightness profile are $\left(x_{m}, y_{m}\right)=(687$ th pixel, 616th pixel), $\left(\Gamma_{x}, \Gamma_{y}\right)=(1288$ pixel, 1187 pixel $)$, and $c_{o}$ $=-0.135$ (dimensionless). The measured values of $(t, V)$ in units of $\mathrm{ms}$ and $\mathrm{ml}$ were $(930,2.7),(980,2.7),(250,3.0),(320$, $3.0)$. . , for which the value $c$ were determined by Lorentzian fitting to be, respectively, $131,114,180,230, \ldots$. The corresponding densities of FNPs were measured to be 10, 10, 50, 50, $\ldots \mu \mathrm{g} / \mathrm{ml}$. From these data we obtained the value of $a$ by using a linear fitting as in Fig. 1(c), and the value was $208 \mu \mathrm{g} / \mathrm{ms}$.

\subsection{Quantification of the Density of Nanoparticles}

The estimate of the line density of nanoparticles in the primo vessels was also a multistep process. The adjacent background intensity, which was due to tissue autoflurescence, was subtracted. The mass of the nanoparticles in the entire reference suspension, $m$, is given by $m=\rho V=a c / t$, where $\rho=$ density of nanoparticles, $V=$ volume, $a$ and $c$ are previously defined. The nanoparticle density of the reference suspension is the volume density (mass over volume). Because we needed a relationship between the surface density of nanoparticles and the fluorescence intensity, we calculated the surface density, defined as the entire nanoparticle mass over the surface area of the reference suspension. The surface density is $\sigma=m / A=a c /(t A)$, where $A$ is the upper surface area of the reference suspension. Then, the nanoparticle mass in a primo vessel is given by $m_{p}$ $=\int \sigma d S=a \int c d S /(A t)$, with the integral being a surface integrand and the line density is $m_{p} / l$, where $l$ is the length of the primo vessel. For the integration, we first calculated $c(x, y)$ from Eq. (1) for the Lorentzian fitting. The calculated nanoparticle line densities are given in Tables 1 and 2 .

\section{Discussion}

The primo vessels floating in the CSF of the fourth ventricle of the brain and in the subarachnoid space of the spinal cord of rats showed strong fluorescence due to the FNPs that had been injected into the lateral ventricle of the rat. The FNPs in the CSF penetrated the primo vessels through pores in the wrapping membrane and were trapped in fibers of the primo vessel. The presence of such pores was revealed using electron microscopic images of the primo vessel, ${ }^{36}$ and a more detailed structure of the pore was only recently obtained with the help of $x$ ray microscopy ${ }^{37}$ Such a fenestrated structure of the primo vessel is distinctively different from the structure of a blood vessel and is the reason the primo vessel absorbed FNPs while the accompanying blood vessel did not, as shown in Fig. 3(c).

The concentrations of the FNPs in the primo vessels were evaluated using fluorescence intensities. The line density in the primo vessel of the subarachnoid space was about 3 times higher than that of the primo vessel in the fourth ventricle. We do not understand the mechanism behind this variation or behind the variations among subject animals. The data obtained in this work may be useful for estimating contrast and for planning experiments for MRI or CT imaging of primo vessels in the brain or in the spinal cord.
The significances of the current work are multifold. It showed that nanoparticles injected into the lateral ventricle flowed with the CSF stream and were absorbed by the primo vessel in other parts of the brain and in the spinal cord far from the injection point. While the primo vessels in the fourth ventricle and in the central canal of the spinal cord were observed as we had planned, the observation of a primo vessel alongside a blood vessel in the subarachnoid space of the spinal cord was unexpected. A primo vessel alongside a blood vessel is so hard to detect by using other methods that the current work is the first such finding, and a surprising result. Furthermore, this discovery provides a critical stepping stone to search for a large network of primo vessels by tracking primo vessels alongside blood vessels.

The preferential absorption of nanoparticles by the primo vessels compared to other tissues such as surrounding ventricular walls or blood vessels was expected as mentioned in the first paragraph of this section. This preferential absorption is an essential factor in detecting a primo vessel in the brain without surgery by using MRI or CT imaging. The data obtained in the present work will be useful in estimating contrast and in designing protocols for MRI or CT imaging of primo vessels in the brain or the spinal cord. Once this imaging technique is completely developed, it will be useful for diagnosis of various brain diseases such as Alzheimer's disease.

\section{Conclusion}

Injection of fluorescent nanoparticles into the lateral ventricle of a rat brain was performed, for the first time, in order to visualize the primo vessels in the brain. We found that the fluorescent nanoparticles were preferentially absorbed by a primo vessel floating in the CSF of the fourth ventricle and by a primo vessel lying alongside a blood vessel in the subarachnoid space of the spinal cord. By using a calibration with reference suspension and a Lorentzian model fitting, we were able to calculate the line densities of the absorbed nanoparticles. They were 0.5 \pm 0.5 and $1.3 \pm 0.6 \mathrm{ng} / \mathrm{mm}$ in the primo vessels in the fourth ventricle and the spinal cord, respectively. The current work proved that nanoparticles injected into CSF were preferentially absorbed so that the contrast necessary to detect a primo vessel in the brain or the spinal cord with MRI or CT imaging was attainable by this injection technique. Imaging of primo vessels in the brain can provide a new diagnostic tool for various brain diseases such as Alzheimer's disease, brain tumors, strokes, and others.

\section{Acknowledgments}

This research was supported by the Pilot Project of the Advanced Institutes of Convergence Technology, Seoul National University, and by the Association of Korean Oriental Medicine.

\section{References}

1. B. Kim, “On Kyungrak System," J. Acad. Med. Sci. DPR Korea 90, 1-41 (1963).

2. B. Kim, "The Kyungrak system," J. of Jo. Sun Medicine 108, 1-38 (1965).

3. K. S. Soh, "Bonghan circulatory system as an extension of acupuncture meridians," J. Acupuncture and Meridian Studies 2(2), 93-106 (2009). 
4. S. Fujiwara, "Bonghan theory' morphological studies," Iagku no Ayumi 60, 567-577 (1967).

5. B. C. Lee, K. Y. Baik, H. M. Johng, T. J. Nam, J. Lee, B. Sung, C. Choi, W. H. Park, E. S. Park, and D. H. Park, "Acridine orange staining method to reveal the characteristic features of an intravascular threadlike structure," Anat. Rec. B: New Anat. 278(1), 27-30 (2004).

6. B. C. Lee and K. S. Soh, "Contrast-enhancing optical method to observe a Bonghan duct floating inside a lymph vessel of a rabbit," Lymphology 41(4), 178-185 (2008).

7. C. Lee, S. K. Seol, B. C. Lee, Y. K. Hong, J. H. Je, and K. S. Soh, "Alcian blue staining method to visualize bonghan threads inside large caliber lymphatic vessels and X-ray microtomography to reveal their microchannels," Lymphat. Res. Biol. 4(4), 181-190 (2006).

8. B. C. Lee, H. B. Kim, B. Sung, K. W. Kim, J. Sohn, B. Son, B. J. Chang, and K. S. Soh, "Network of endocardial vessels," Cardiology 118(1), 1-7 (2011).

9. H. S. Shin, H. M. Johng, B. C. Lee, S. I. Cho, K. S. Soh, K. Y. Baik, and J. S. Yoo, "Feulgen reaction study of novel threadlike structures (Bonghan ducts) on the surfaces of mammalian organs," Anat. Rec. B: New Anat. 284(1), 35-40 (2005).

10. B. C. Lee, S. Kim, and K. S. Soh, "Novel anatomic structures in the brain and the spinal cord of a rabbit that may belong to the Bonghan system of potential acupuncture meridians," J. Acupuncture and Meridian Studies 1(1), 29-35 (2008).

11. J. Lim, "Visualization of the primo vascular system in spinal cord and brain with fluorescent nano particles," PhD Thesis, Seoul National University, Seoul (2011).

12. M. S. Kim, B. Sung, B. C. Lee, J. S. Yoo, S. H. Lee, Y. J. Kim, K. W. Kim, and K. S. Soh, "Measurement of flow speed in the channels of novel threadlike structures on the surfaces of mammalian organs," Naturwiss. 95(2), 117-124 (2008).

13. J. Kim, V. Ogay, B. Lee, M. Kim, I. Lim, H. Woo, H. Park, J. Kehr, and K. Soh, "Catecholamine-producing novel endocrine organ: Bonghan system," Med. Acupuncture 20(2), 97-102 (2008).

14. S. J. Lee, B. C. Lee, C. H. Nam, W. C. Lee, S. U. Jhang, H. S. Park, and K. S. Soh, "Proteomic analysis for tissues and liquid from bonghan ducts on rabbit intestinal surfaces," J. Acupuncture and Meridian Studies 1(2), 97-109 (2008).

15. J. S. Yoo, H. B. Kim, N. Won, J. Bang, S. Kim, S. Ahn, B. C. Lee, and K. S. Soh, "Evidence for an additional metastatic route: In vivo imaging of cancer cells in the primo-vascular system around tumors and organs," Mol. Imaging Biol. 13(3), 471-480 (2010).

16. J. Yoo, M. Ayati, H. Kim, W. Zhang, and K. Soh, "Characterization of the primo-vascular system in the abdominal cavity of the lung cancer mouse model and its differences from the lymphatic system," PLoS ONE 5(4), e9940 (2010).

17. K. Y. Baik, V. Ogay, S. C. Jeoung, and K. S. Soh, "Visualization of Bonghan microcells by electron and atomic force microscopy," J. Acupuncture and Meridian Studies 2(2), 124-129 (2009).

18. M. Kucia, M. Wysoczynski, J. Ratajczak, and M. Ratajczak, "Identification of very small embryonic like (VSEL) stem cells in bone marrow," Cell Tissue Res. 331(1), 125-134 (2008).

19. M. H. Cho, J. T. Kwon, S. K. Hwang, H. Jin, D. S. Kim, A. Mina-Tehrani, H. J. Yoon, M. Chop, T. J. Yoon, D. Y. Han, Y. W. Kang, B. I. Yoon, and J. K. Lee, "Body distribution of inhaled fluorescent magnetic nanoparticles in mice," J. Occup. Health 50(1), 1-6 (2008).
20. A. Louie, "Multimodality imaging probes: design and challenges," Chem. Rev. 110(5), 3146-3195 (2010).

21. I. o. L. A. R. C. o. L. Sciences, Guide for the Care and Use of Laboratory Animals, National Academy Press, Washington, DC (1996).

22. T. J. Yoon, J. S. Kim, B. G. Kim, K. N. Yu, M. H. Cho, and J. K. Lee, "Multifunctional nanoparticles possessing a "magnetic motor effect" for drug or gene delivery," Angew. Chem., Int. Ed. Engl. 44(7), 1068-1071 (2005).

23. J. S. Yoo, H. M. Johng, T. J. Yoon, H. S. Shin, B. C. Lee, C. Lee, B. S. Ahn, D. I. Kang, J. K. Lee, and K. S. Soh, "In vivo fluorescence imaging of threadlike tissues (Bonghan ducts) inside lymphatic vessels with nanoparticles," Curr. Appl. Phys. 7(4), 342-348 (2007).

24. E. Peli, "Contrast in complex images," J. Opt. Soc. Am. A 7(10), 2032 2040 (1990)

25. E. Greene, Anatomy of the Rat, Hafner Pub. Co., New York (1963).

26. A. Elsaesser, A. Taylor, G. S. de Yanes, G. McKerr, E. M. Kim, E. O'Hare, and C. V. Howard, "Quantification of nanoparticle uptake by cells using microscopical and analytical techniques," Nanomedicine 5(9), 1447-1457 (2010).

27. V. Ntziachristos, C. Bremer, and R. Weissleder, "Fluorescence imaging with near-infrared light: new technological advances that enable in vivo molecular imaging," Eur. Radiol. 13(1), 195-208 (2003).

28. S. Kascáková, B. Kruijt, H. S. de Bruijn, A. van der Ploeg-van den Heuvel, D. J. Robinson, H. J. C. M. Sterenborg, and A. Amelink, " $E x$ vivo quantification of mTHPC concentration in tissue: Influence of chemical extraction on the optical properties," J. Photochem. Photobiol., B 91(2-3), 99-107 (2008).

29. K. R. Diamond, P. P. Malysz, J. E. Hayward, and M. S. Patterson, "Quantification of fluorophore concentration in vivo using two simple fluorescence-based measurement techniques," J. Biomed. Opt. 10(2), 024007 (2005).

30. K. R. Diamond, M. S. Patterson, and T. J. Farrell, "Quantification of fluorophore concentration in tissue-simulating media by fluorescence measurements with a single optical fiber," Appl. Opt. 42(13), 2436$2442(2003)$.

31. M. A. Model and J. K. Burkhardt, "A standard for calibration and shading correction of a fluorescence microscope," Cytometry 44(4), 309-316 (2001).

32. M. A. Model, "Intensity calibration and shading correction for fluorescence microscopes," in Current Protocols in Cytometry, John Wiley \& Sons, New York (2001).

33. Likar, Maintz, Viergever and Pernuš, "Retrospective shading correction based on entropy minimization," J. Microsc. 197(3), 285-295 (2000).

34. A. H. Lettington, M. P. Rollason, S. Tzimopoulou, and E. Boukouvala, "Image restoration using a two-dimensional Lorentzian probability model," J. Mod. Opt. 47(5), 931-938 (2000).

35. J. Condon, "Errors in elliptical Gaussian fits," Publ. Astron. Soc. Pac. 109, 166-172 (1997).

36. B. C. Lee, J. S. Yoo, V. Ogay, K. W. Kim, H. Dobberstein, K. S. Soh, and B. S. Chang, "Electron microscopic study of novel threadlike structures on the surfaces of mammalian organs," Microsc. Res. Tech. 70(1), 34-43 (2007).

37. M. Kim, S. Oh, J. Lim, and S. Han, "Phase contrast x-ray microscopy study of rabbit primo vessels," Appl. Phys. Lett. 97, 213703 (2010). 